\title{
HALLAZGO DE FRAGMENTOS CERÁMICOS EN LA MESETA BELLA VISTA (CAMPO VOLCÁNICO PALI AIKE, PROVINCIA DE SANTA CRUZ, ARGENTINA)
}

NATALIA A. CIRIGLIANOa

\section{RESUMEN}

Los trabajos de campo arqueológicos desarrollados en el área de Meseta Bella Vista, localizada en el sector centro-oeste del campo volcánico Pali Aike, provincia de Santa Cruz, Argentina, permitieron detectar una concentración en superficie de 11 fragmentos cerámicos. En este artículo se presentan los resultados de los análisis macroscópicos de los tiestos y microscópicos de las pastas y los sedimentos locales. Los resultados obtenidos apoyan la hipótesis que plantea la obtención de recipientes cerámicos en espacios localizados más al norte, posiblemente mediante interacción social, y respaldan la existencia de estrategias de conservación y transporte ocasional de vasijas entre los cazadores-recolectores de Patagonia extremo sur continental.

PALABRAS CLAVE: cazadores-recolectores, cerámica, Patagonia extremo sur continental.

$$
\begin{gathered}
\text { POTTERY FRAGMENTS FOUND IN THE BELLA VISTA PLATEAU } \\
\text { (PALI AIKE VOLCANIC FIELD, SANTA CRUZ PROVINCE, ARGENTINA) }
\end{gathered}
$$

\section{ABSTRACT}

The archaeological field work carried out in the Bella Vista Plateau area, located in the centerwest of Pali Aike Volcanic Field, Santa Cruz Province, Argentina, led to the detection of a surface concentration of 11 ceramic fragments. This paper provides the results of macroscopic data on pottery fragments and microscopic analysis of pastes and local sediments. The results support the hypothesis that ceramic containers were acquired in the North, possibly through social interaction. The data also supports the existence of curation strategies and occasional transport of vessels among hunter-gatherers in the Southernmost part of continental Patagonia.

KEY WORDS: hunter-gatherers, ceramic, Southernmost part of continental Patagonia.

a Consejo Nacional de Investigaciones Científicas y Técnicas, Instituto Multidisciplinario de Historia y Ciencias Humanas/ Universidad de Buenos Aires, Facultad de Filosofía y Letras. Saavedra 15, 5to piso, C.A.B.A. (C.P. 1083) / Puán 480, C.A.B.A. (C.P. 1406), Argentina. $\$ naticirigliano2015@gmail.com 


\section{INTRODUCCIÓN}

El registro cerámico del área de Patagonia sur es poco frecuente. No obstante, se pueden reconocer sectores con mayor abundancia de elementos cerámicos que otros. Entre éstos, cabe señalar los espacios localizados al NE costero y NW pericordillerano de la provincia de Santa Cruz, incluyendo la porción chilena, al este de la cordillera de los Andes (Cassiodoro \& Tchilinguirian, 2007; Chaile et al. 2020; Cirigliano et al. 2019 y citas allí incluidas; Mena \& Jackson, 1991; Roumec et al. 2020; Zilio et al. 2018; entre otros). La frecuencia de este registro es menor en los espacios localizados al sur del río Santa Cruz (Martinic \& Prieto, 1998), donde sólo se identificaron elementos cerámicos en muy pocos sectores. Uno de estos espacios corresponde a la localidad arqueológica de San Gregorio donde se registraron tiestos con superficies lisas, sin pintura o decoración y de espesores variables (Martinic, 1984; Massone, 1984). Asimismo, se reconocieron ítems cerámicos en la colección alojada en Estancia Los Pozos, próxima al río Gallegos (Gómez Otero \& Barbería, 1982). Aunque este conjunto se conforma por tiestos y otros objetos cerámicos mayormente de dudosa procedencia ${ }^{1}$, algunos elementos, tales como una bola de pequeñas dimensiones cocida en atmósfera reductora y un cuerpo con base de pipa, habrían sido recuperados en esa estancia y en el área de Cabo Buen Tiempo respectivamente. También se reconocieron pipas o fragmentos de pipas cerámicas de manufactura indígena en los sitios arqueológicos San Gregorio y Dinamarquero; y en la colección etnográfica de Schythe, obtenida ésta por medio de intercambios a pedido expreso del gobernador con indígenas en la Colonia de Punta Arenas (Martinic, 1993-94; Martinic \& Prieto, 1998).

Es significativo destacar que algunos investigadores del área de Patagonia sur propusieron sobre la base de los análisis microscópicos de las pastas y los sedimentos posibles espacios destinados a la manufactura de recipientes cerámicos. Aunque este es el caso de la cuenca Salitroso-PosadasPueyrredón, localizada al NW de la provincia de
Santa Cruz (Cassiodoro \& Tchilinguirian, 2007), la producción de recipientes cerámicos no parece haber ocurrido más al sur. Por el contrario, el hallazgo ocasional de tiestos al sur del área mencionada fue explicado a partir de la obtención de contenedores por medio del intercambio o la interacción con otros grupos situados más al norte (Cirigliano et al. 2019; Martinic \& Prieto, 1998) 2 .

En líneas generales, el registro cerámico de Patagonia sur fue temporalmente asignado a los últimos 1.500 años (Chaile et al. 2020 y citas allí incluidas; Cirigliano et al. 2019; Gradin, 2000; Mena \& Lucero, 2004; Moreno \& Videla, 2008; entre otros) y fue frecuentemente asociado a momentos históricos en su extremo más meridional (Bird, 1988; Martinic, 1984; Martinic \& Prieto, 1998). Según Martinic y Prieto (1998), los recipientes cerámicos habrían sido adquiridos a partir del intercambio intercultural durante momentos ecuestres. Para estos investigadores, el caballo posibilitó los intercambios interétnicos y el acceso a la tecnología cerámica de forma ocasional durante los viajes anuales, situación que explicaría la baja frecuencia de este tipo de material en el registro arqueológico de Patagonia extremo sur.

Recientemente, a raíz de las nuevas prospecciones realizadas en el área del interfluvio Gallegos-Chico, se notificó sobre el hallazgo de 11 fragmentos cerámicos en la Meseta Bella Vista, sector centro-oeste del campo volcánico Pali Aike (CVPA), provincia de Santa Cruz, Argentina (Borrazzo et al. 2019). De manera preliminar y desde un abordaje macroscópico, Borrazzo y colaboradores (2019) señalaron la existencia de un recipiente cerámico de borde evertido y forma cerrada con superficies pulidas y decoradas. En vista de que la información sobre este registro fue parcialmente publicada, el objetivo de este trabajo es caracterizar con mayor detalle al conjunto cerámico desde un abordaje macroscópico y microscópico. Esto permitirá conocer la composición petrográfica de las pastas y comprobar la existencia de un único recipiente. Asimismo, se evaluará a partir del estudio de una muestra de sedimento la posibilidad de la manufactura local del contenedor.

1 En la colección se reconocieron fragmentos de bordes de vasijas y tiestos de coloración gris oscuro con superficies alisadas o pulidas de manera burda.

2 No obstante, Martinic y Prieto (1998) y Martinic (1995) plantean que la producción de alfarería para la confección de pipas o con fines lúdicos u ornamentales se habría desarrollado en Patagonia sur durante momentos históricos muy tardíos (a partir de mediados del siglo XIX) (ver también Priegue, 2007, pp. 27, 55 y 69 sobre el uso de barro cocido y no cocido vinculado con actividades lúdicas infantiles). 

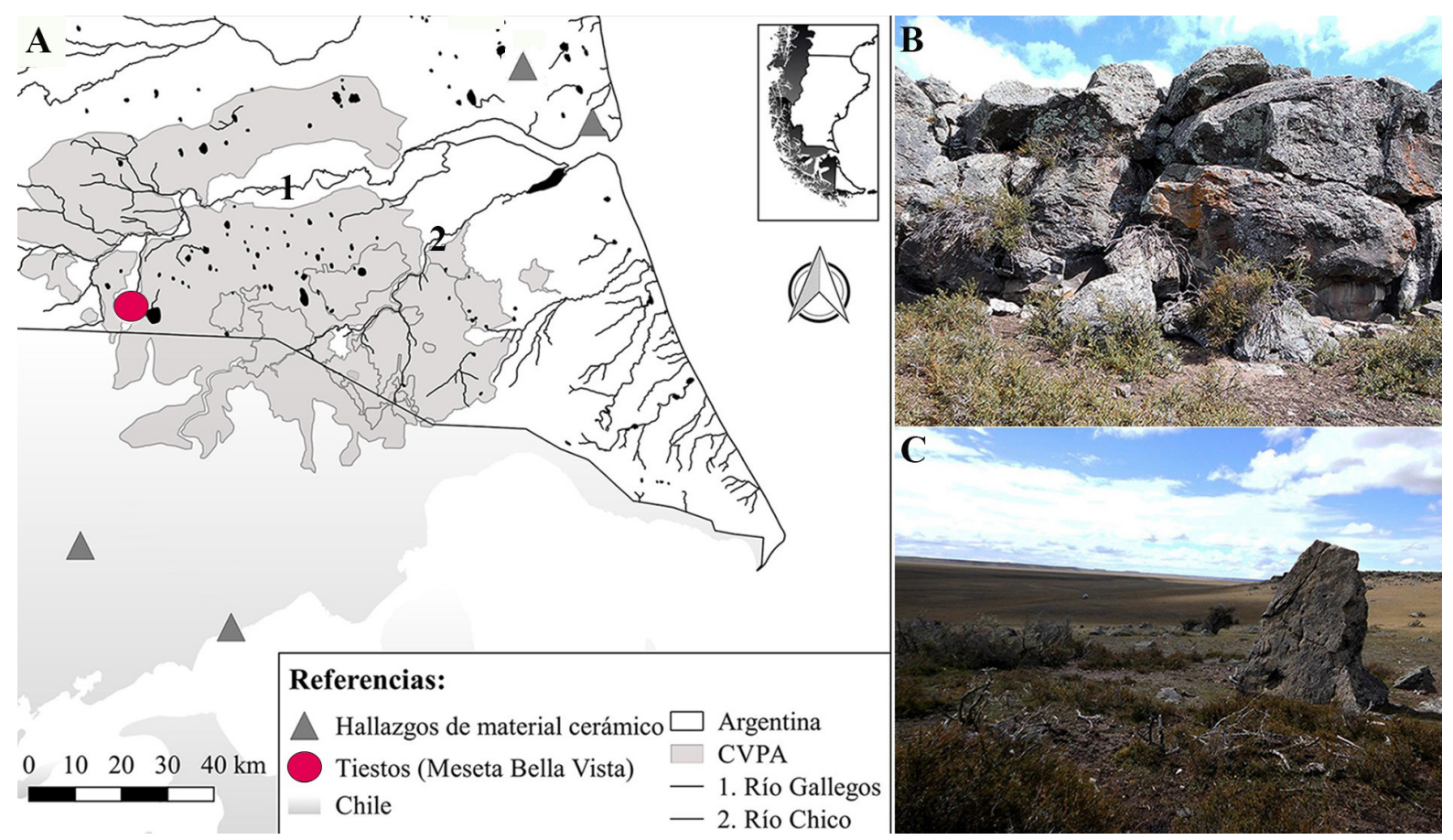

Fig. 1. Área de estudio. Referencias: hallazgos cerámicos en Patagonia extremo sur continental (A); lugar del hallazgo de los tiestos en MBV (B); vista del entorno (C).

\section{ÁREA DE ESTUDIO}

El CVPA se localiza en el extremo sur de Patagonia continental, cubriendo sectores tanto en Chile como en Argentina (Corbella, 2002; D'Orazio et al. 2000). Las investigaciones arqueológicas establecieron una secuencia de ocupación para el CVPA entre ca. 11.000 años AP y momentos históricos (e.g. Bird, 1988; Gómez Otero, 198990; Martin \& San Román, 2010; Massone, 1981, 1989-90; Massone \& Hidalgo, 1981), aunque en los espacios más cercanos al área bajo estudio, los antecedentes muestran ocupaciones humanas a partir del Holoceno medio, siendo éstas más frecuentes durante el Holoceno tardío (Banegas et al. 2014; Borrero et al. 2013; Campan et al. 2007; Charlin et al. 2018; Gómez Otero, 1987; L'Heureux et al. 2021a; entre otros).

La Meseta Bella Vista (en adelante MBV) se localiza en el centro-oeste del CVPA, provincia de Santa Cruz, Argentina (Fig. 1A), y a menos de $5 \mathrm{~km}$ de la laguna Potrok Aike, fuente de agua permanente y confiable desde hace al menos 16.000 años (Borrero et al. 2013). En el sector se reconocen distintas geoformas (mesetas basálticas, terrazas, planicies aluviales), cotas altitudinales bajas (100 a $250 \mathrm{~m}$ ) y fuentes de agua estacionales (arroyos y bajos lagunares). La meseta, correspondiente a la unidad volcanitas Bella Vista, presenta aleros, cuevas de pequeñas dimensiones y paredones (Manzi et al. 2019; Panza \& Sacomani, 2015). Ésta se encuentra constituida por basaltos olivínicos y volcanitas ultramáficas olivínicas (Panza \& Sacomani, 2015). Inmediatamente al oeste de la misma se localiza el chorrillo Carlota (ex río Robles), de régimen efímero, que labró su cauce disectando los depósitos del segundo nivel de terrazas fluviales del río Gallegos. En estos asomos se identifican basaltos, andesitas, brechas riolíticas, metamorfitas, granodioritas, granitos, pelitas y areniscas (Panza \& Sacomani, 2015). Los relevamientos mostraron la existencia natural de pigmentos -hematita- (Oriolo et al. 2019) y de rocas de calidades aptas para la talla.

Los fragmentos cerámicos aquí analizados proceden de una recolección selectiva $(2 \mathrm{~m}$ por 2 $\mathrm{m})$ realizada al pie de un paredón en el marco de tareas de exploración (Fig. 1B y C). Allí también se recuperó una lasca angular confeccionada sobre 


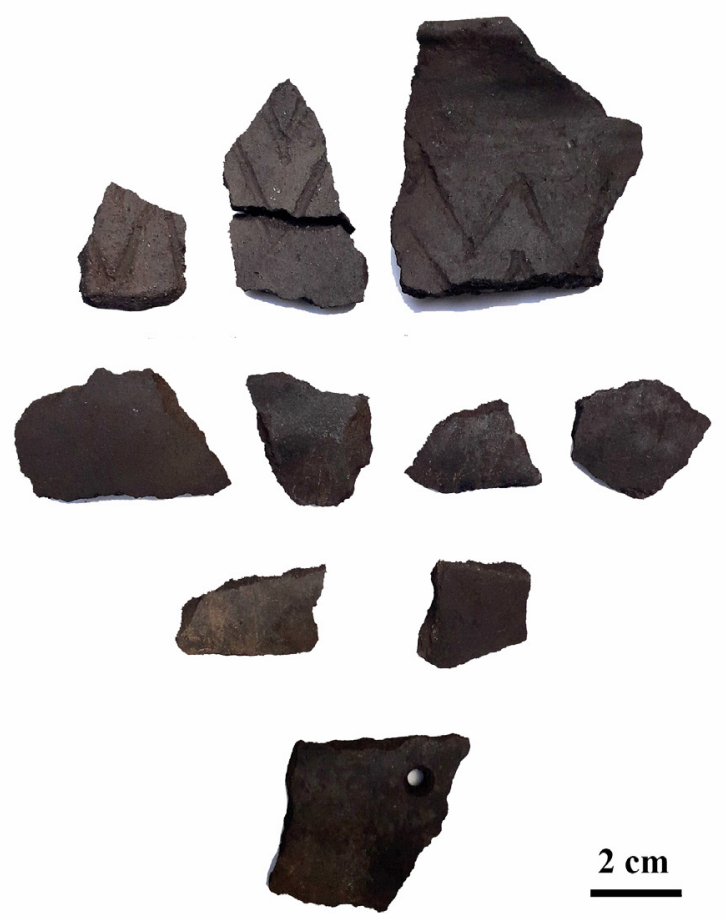

Fig. 2. Fragmentos cerámicos hallados en MBV.

materia prima local (Roca de Grano fino Oscura tipo Potrok Aike). La recolección se efectuó en superficie y al aire libre. En sus proximidades se reconoció gran cantidad de artefactos líticos, algunos de ellos de tamaño considerable (e.g. yunque, yunque-mano con pigmento adherido). Asimismo, en el área de MBV se registró abundante arte rupestre que incluye motivos grabados (e.g. camélidos de vientre abultado o sobredimensionado) y pintados (e.g. manos, tridígitos, trazos quebrados), que podría corresponder al Holoceno medio y tardio respectivamente (Manzi \& Carballo Marina, 2012; Manzi et al. 2019). Cabe destacar que algunos motivos de grandes dimensiones asociados con una greca podrían corresponder a momentos históricos (Manzi et al. 2019). Por otra parte, un sondeo realizado en el sitio Bella Vista 1 arrojó una datación de ca. 1.400 años AP (L'Heureux et al. 2021a).

\section{METODOLOGÍA}

El análisis macroscópico fue abordado con el objetivo de conocer las características del material cerámico siguiendo los lineamientos metodológicos propuestos por Powers (1953), la Primera Convención
Nacional de Antropología (1966), Balfet y otros (1992), Orton y colaboradores (1997), entre otros. Se realizaron cortes delgados de dos tiestos, con y sin decoración, que no remontan entre sí para conocer las características de las pastas y corroborar la existencia de un único recipiente. Para el análisis de los cortes se utilizó un microscopio de polarización petrográfico LEICA con binoculares de distintos aumentos - $4 \mathrm{x}$ y $10 \mathrm{x}$ - Para conocer la característica de la pasta (porcentaje de inclusiones, matriz y poros) se realizó un conteo de 400 puntos equidistantes entre sí, a la vez que se comparó con cuadros visuales que estiman proporciones. Se evaluó el tipo y el tamaño de las inclusiones (mineralogía de la fracción arena). Los tipos de inclusiones se consignaron como elementos mayoritarios ( $\geq 25 \%$ ), minoritarios $(<25 \%$ a $\geq 5 \%$ ) y accesorios $(<5 \%)$. Asimismo, para conocer la composición de los sedimentos locales y compararla con la constitución de las pastas, se tomó una muestra en un sector próximo al hallazgo. La muestra fue previamente tratada por la Dra. Ivana Ozán que removió la materia orgánica y los clastos de tamaños muy pequeños (limos y arcillas) para su posterior montaje en una lámina de vidrio. 



\section{$1 \mathrm{~cm}$}
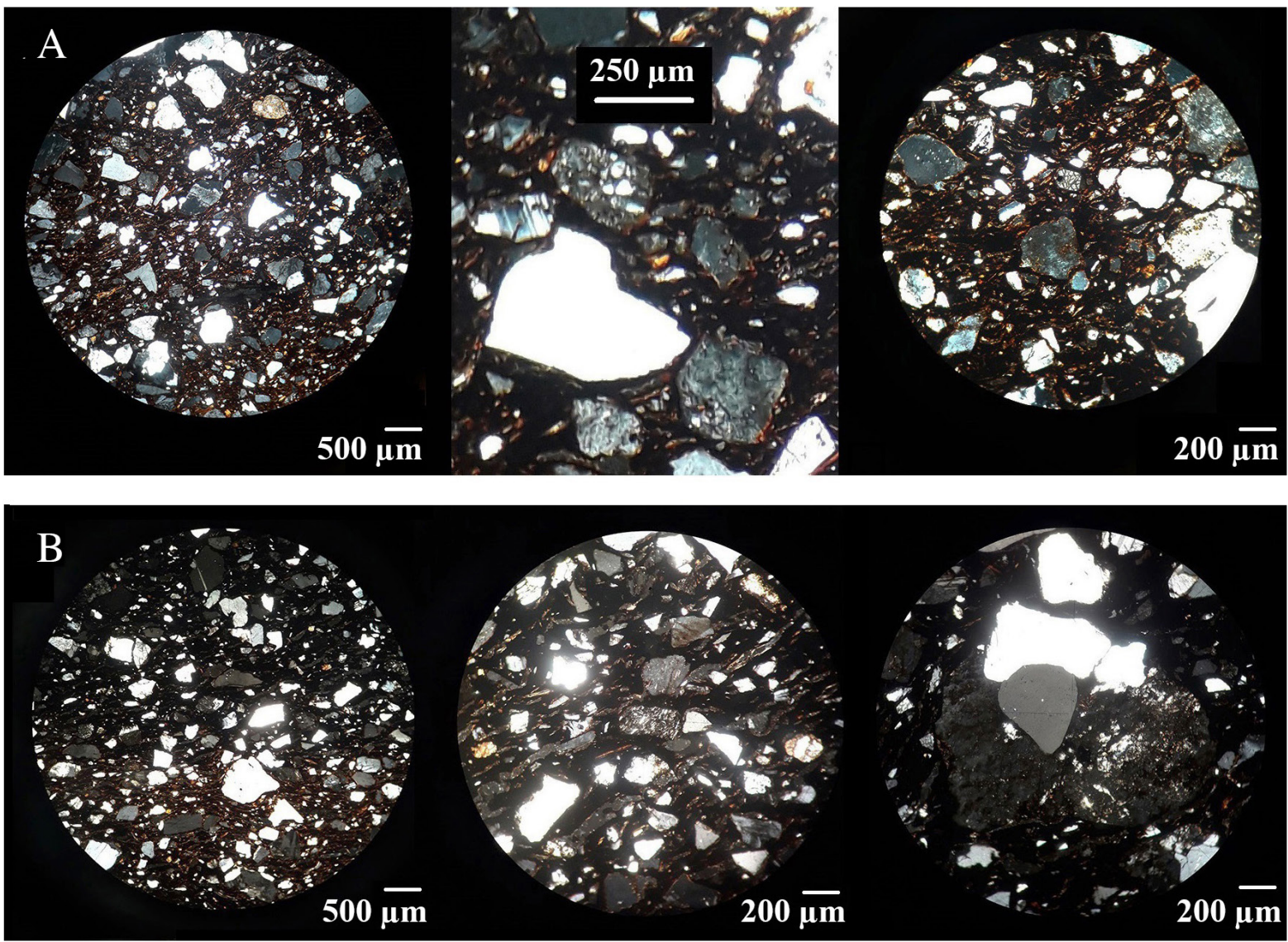

Fig. 3. Tiestos y microfotografías de las secciones delgadas.

Referencias: Muestra 2 (A) y Muestra 11 (B). 


\section{RESULTADOS}

\section{Análisis macroscópico de los tiestos}

La muestra se conforma de 11 tiestos. Diez corresponden a fragmentos de cuerpo y uno a borde con labio convexo o redondeado (Fig. 2). Se registró al menos un punto de inflexión entre borde y cuerpo. Los espesores de los fragmentos, medidos en los sectores de la pieza sin descascaramiento de las superficies, se encuentran comprendidos entre 6,5 y $9,5 \mathrm{~mm}$.

Los extremos fragmentados de los tiestos son en todos los casos angulosos, lo que permite inferir la escasez de transporte por agentes naturales (Powers, 1953; Skibo, 1987). La tarea de remontaje facilitó el ensamblaje de dos tiestos (Fig. 2). Con respecto al tratamiento de la superficie (sensu Balfet et al. 1992), los fragmentos presentan pulido y únicamente cuatro registran decoración. La modificación de la superficie se encuentra bajo la forma de incisiones $(n=4)$. Por la profundidad y el arrastre de la arcilla se considera que la decoración del recipiente se realizó cuando la pieza se encontraba aún fresca y blanda. Se reconocen únicamente motivos geométricos: líneas quebradas o en zig-zag (al menos dos hileras), confeccionadas, en el caso identificable, en forma horizontal al eje técnico de la pieza. Entre los tiestos sin decorar se registró un agujero de reparación.

La pasta en los bordes fracturados es de coloración oscura, lo que posiblemente se deba a una cocción pobre en oxígeno (Orton et al. 1997). La mayoría de los fragmentos exhibe superficies tiznadas o sustancias ennegrecidas o carbonosas adheridas. El color de la superficie externa, considerando los sectores tiznados y no tiznados, corresponde a la paleta 10YR con variaciones de valor -luminosidad- $y$ croma -saturación- (Munsell Color, 1992).

El diámetro de la boca del recipiente no pudo ser consignado debido al pequeño tamaño del borde del fragmento ( $\leq 5 \%$ del diámetro a estimar) y a su escaso arco, lo que podría resultar en estimaciones imprecisas de esta variable.

\section{Análisis microscópico de las pastas}

Como ya se señaló, para realizar la lámina delgada se escogió un tiesto con incisiones (Muestra 2) y otro sin incisiones (Muestra 11), que además presentaban tonalidades disímiles en su superficie (Fig. 3). El análisis microscópico muestra una proporción de matriz ( 35\%), inclusiones no plásticas $(\sim 45 \%)$ y poros $(\sim 20 \%)$ prácticamente equivalente en ambas pastas.

Con respecto a la matriz arcillosa, se registran importantes cantidades de hierro que le dan una coloración rojiza a la pasta. También se hacen evidentes cambios en la coloración de la misma. En este sentido, la pasta de la Muestra 2 presenta una delgada línea de coloración castaña (10 YR 4/4 dark yellowish brown) en un extremo del corte -superficie externa del tiesto-, mientras que, en el resto de la muestra, la coloración es más oscura (10 YR 2/1 black). La Muestra 11 registra dos franjas de grosor similar. Una de coloración 10 YR 3/3 (dark brown) próxima a la superficie externa y otra 10 YR 2/1 (black) en la zona restante. Los cambios detectados en la coloración de la pasta podrían deberse a variaciones en la atmósfera de cocción o a la absorción de sustancias durante el uso del recipiente, entre otros factores (Orton et al. 1997; Shepard, 1956).

La composición mineralógica también es similar en ambas muestras. Entre los elementos mayoritarios $(\geq 25 \%)$ se reconoce únicamente feldespato potásico, mientras que entre los elementos minoritarios $<25 \%$ $a \geq 5 \%$ ) se identifica cuarzo, litoclasto y feldespato plagioclasa. Entre los litoclastos de ambas muestras se registran fragmentos de rocas plutónicas ácidas y rocas volcánicas básicas y ácidas o mesosilícicas. Por su parte, los elementos accesorios $(<5 \%)$ corresponden a minerales opacos, piroxeno, anfíbol y biotita (Fig. 3). Las inclusiones no se encuentran bien seleccionadas, ya que presentan tamaños variables de la fracción arena (fina, media, gruesa y muy gruesa). No obstante, en ambas muestras, la textura media/fina es la predominante.

\section{Análisis de los sedimentos}

La muestra de sedimento se obtuvo a $25 \mathrm{~m}$ del sector donde se recuperó el conjunto cerámico y es representativa de la ubicua cubierta eólica pobremente edafizada del área (L'Heureux et al. 2021b). Entre los elementos mayoritarios ( $\geq 25 \%$ ) se registran fragmentos líticos y feldespato plagioclasa (Fig. 4). Entre los litoclastos dominan las rocas volcánicas ácidas, mesosilícicas y básicas, registrándose escasas rocas con bajo grado de metamorfismo. El resto de los minerales se encuentra en muy bajo porcentaje y corresponden a elementos accesorios ( $<5 \%)$. Entre éstos se identifican minerales opacos, cuarzo, piroxeno, anfíbol, feldespato potásico, epidota, olivino y apatito. El sedimento registra también óxido de hierro. Cabe destacar que los litoclastos y minerales identificados son consistentes con la geología local (ver supra, Oriolo et al. 2019; Panza \& Sacomani, 2015). 


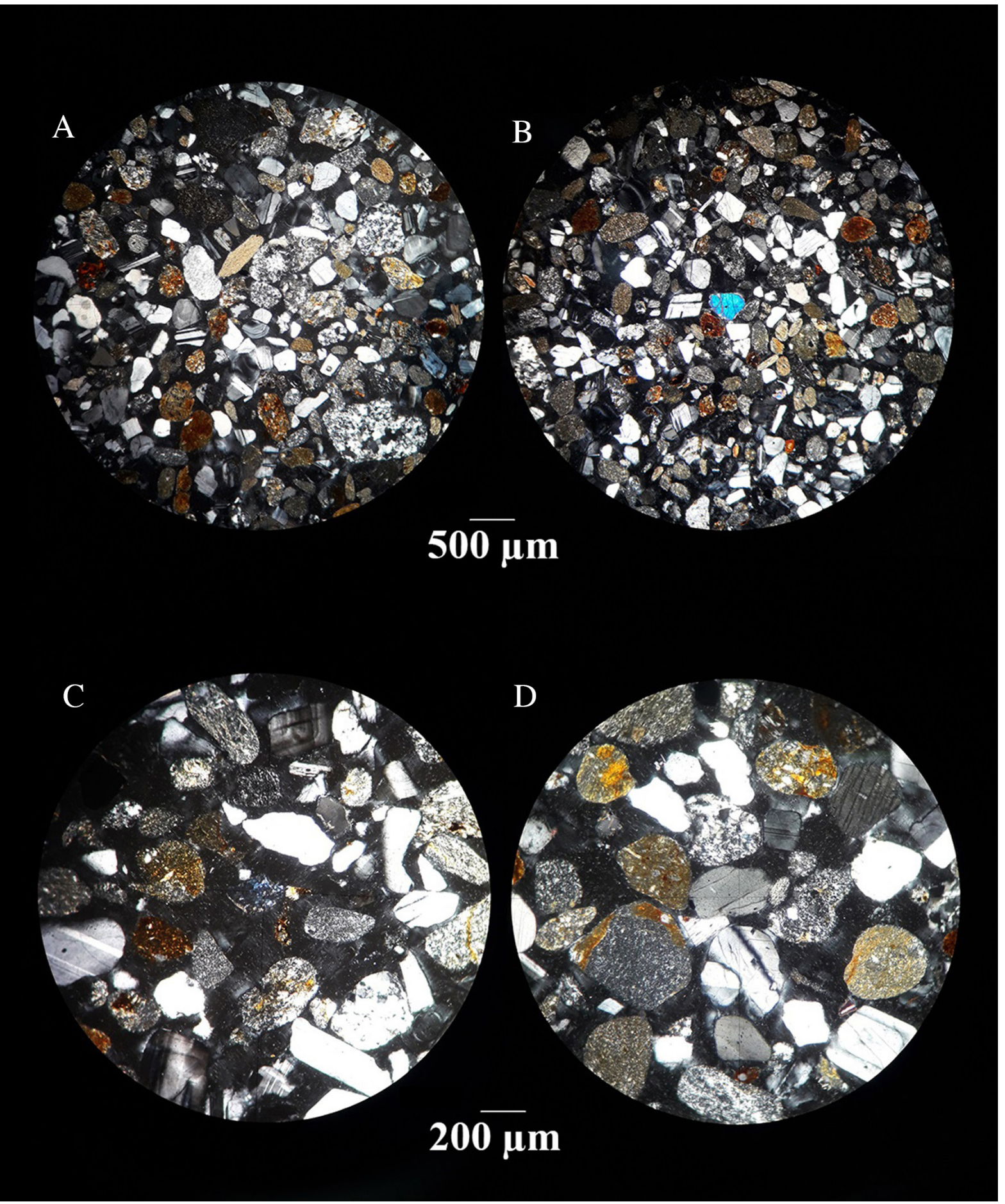

Fig. 4. Microfotografías de la muestra de sedimento. Referencias: litoclastos y cristales de feldespato plagioclasa (A); en el centro del campo de visión: olivino (B), epidota (C) y apatito (D). 


\section{CONSIDERACIONES GENERALES}

El análisis microscópico de las secciones delgadas de los tiestos apunta a la existencia de un único recipiente cerámico. Se trata de una vasija de forma cerrada y cuello evertido con superficies pulidas y decoradas mediante incisiones de motivos de líneas quebradas o en zig-zag. Sobre la base de los espesores medidos podría tratarse de un contenedor de paredes medianas-gruesas (Frére et al. 2012).

Las diferencias reconocidas entre las pastas cerámicas y la muestra de sedimento no concuerdan con la elaboración local del recipiente. Por el contrario, apuntan a su posible transporte desde algún otro espacio, lo que a su vez se ve reforzado por la escasez del registro cerámico en el área bajo estudio. El reconocimiento de un agujero de reparación en uno de los tiestos sugiere la inversión de energía en la conservación y el mantenimiento del recipiente.

Aunque se desconoce cuál es el lugar de manufactura de la vasija, algunas de las características de la decoración o de la composición de la pasta permiten elaborar hipótesis acerca de su recorrido o procedencia. Tanto la composición mineralógica de las muestras cerámicas de MBV como de los sedimentos, arcillas y cerámicas del área del noroeste de la provincia de Santa Cruz (Cassiodoro \& Tchilinguirian, 2007) son petrográficamente distintas. Tampoco se han registrado motivos de líneas quebradas en recipientes cerámicos de esa área (Cassiodoro et al. 2019). En cambio, la cerámica recuperada en MBV comparte con aquellos tiestos localizados en el noreste costero de la provincia de Santa Cruz, motivos de líneas quebradas o en zig-zag (e.g. Outes, 1904; Roumec et al. 2020; Trola \& Ciampagna, 2011; Zilio et al. 2018). A partir de la información expuesta se considera que el recipiente de MBV podría proceder de algún sector localizado más al norte, en coincidencia con lo sugerido por otros investigadores para la cerámica del área de Patagonia extremo sur (Borrazzo et al. 2019; Martinic \& Prieto, 1998). Cabe destacar que en ambos espacios -NE costero y Meseta Bella Vista- se reconocieron agujeros de reparación (ver Trola \& Ciampagna, 2011; Zilio et al. 2018), lo que podría estar indicando interés por el mantenimiento de los recipientes cerámicos.

Sobre la base del registro arqueológico recuperado se reconoce que el sector de MBV fue recurrentemente utilizado, al menos durante el Holoceno tardío, momento en que se registra una mayor cantidad de ocupaciones humanas en el CVPA. La existencia de artefactos que por su peso y tamaño (e.g. yunque, mano-yunque con pigmentos, recipiente cerámico) son difíciles de transportar podría estar indicando equipamiento (sensu Binford, 1979) y reocupación planificada del área. La gran abundancia de material lítico y de arte rupestre reconocida durante las prospecciones apunta también a un uso intenso y recurrente de este sector del espacio (Borrazzo et al. 2019; Manzi et al. 2019).

Los recipientes cerámicos son pesados y frágiles, y claro está que por las potencialidades que presentaba el equino como medio de transporte, la cerámica fuera asociada a momentos históricos ecuestres en Patagonia extremo sur continental (Martinic \& Prieto, 1998). No obstante lo anterior, el motivo de líneas quebradas o en zig-zag circuló en distintos soportes móviles durante el Holoceno tardío final a escala regional (e.g. Acevedo, 2015; Cassiodoro et al. 2019; Prieto, 1984), encontrándose las dataciones más australes sobre el uso de recipientes cerámicos en ca. 420 y 140 años AP (Cirigliano et al. 2019). Se planteará a futuro trabajos de campo que involucren sondeos en el lugar del hallazgo y estudios de termoluminiscencia o AMS de las sustancias orgánicas adheridas a los tiestos para obtener cronologías vinculadas al contenedor recuperado en MBV.

\section{AGRADECIMIENTOS}

A las familias Clark, Fernández y Kennard por el apoyo brindado durante los trabajos de campo. A mis compañeras de equipo por responder mis consultas y brindar información (Karen Borrazzo, Judith Charlin, Lorena L'Heureux, Liliana Manzi, Ivana Ozán y María Cecilia Pallo). Se agradece a Pablo Leal, Sebastián Oriolo, Ivana Ozán y Valeria Palamarczuk por los comentarios acerca de las muestras aquí analizadas. A Cecilia Pallo y Luis Borrero por la lectura y sugerencias a una versión previa de este trabajo. Por último, quiero agradecer a dos revisores anónimos porque sus observaciones permitieron enriquecer este trabajo. La investigación fue financiada por Proyectos ANPCyT (PICT 20142061, PICT 2019-02106, PICT 2019-01356) y CONICET. 


\section{BIBLIOGRAFÍA}

Acevedo, A. (2015). Hachas grabadas, placas grabadas y comunicación visual suprarregional entre grupos cazadores-recolectores de finales del Holoceno tardío. Relaciones de la Sociedad Argentina de Antropología, XL(2), 589-620.

Balfet, H., Fauver-Berthelot, M. F., y Monzon, S. (1992). Normas para la descripción de vasijas. Centre d'Études Mexicaubes Et Centraméricaines (CEMCA).

Banegas, A., Gómez Otero, J., Goye, D., y Ratto, N. (2014). Cabezales líticos del Holoceno tardío en Patagonia meridional: Diseños y asignación funcional. Magallania, 42(2), 155-174. http://doi.org/10.4067/S071822442014000200009

Binford, L. R. (1979). Organization and formation processes: looking at curated technologies. Journal of Anthropological Research, 35(3), 255-273.

Bird, J. (1988). Travels and Archaeology in South Chile. University of Iowa Press.

Borrazzo, K. B., L'Heureux, L. G., Cirigliano, N. A., Ozán, I. L., Pallo, M. C., Manzi, L. M., y Charlin, J. E. (2019). Arqueología del interfluvio Gallegos-Chico (Santa Cruz, Argentina): nuevas prospecciones. En J. Gómez Otero, A. Svoboda y A. Banegas (Eds.), Arqueología de la Patagonia: el Pasado en las Arenas (pp. 271-282). Altuna Impresores.

Borrero, L., Barberena, R., Charlin, J., y Campan, P. (2013). Geoarqueología y tafonomía en la cuenca de Potrok Aike. En J. Rubin de Rubin y R. da Silva (Eds.), Geoarqueología (pp. 9-24). Pontificia Universidad Católica de Goiás.

Campan, P., Carballo Marina, F., y Manzi, L. (2007). Arqueología de Estancia La Carlota (Campo Volcánico Pali Aike, Argentina). En F. Morello, M. Martinic, A. Prieto y G. Bahamonde (Eds.), Arqueología de Fuego Patagonia. Levantando piedras, desenterrando huesos... y develando arcanos (pp. 687-700). CEQUA.

Cassiodoro, G., y Tchilinguirian, P. (2007). Análisis petrográficos de cerámicas en el noroeste de la provincia de Santa Cruz. En F. Morello, M. Martinic, A. Prieto y G. Bahamonde (Eds.), Arqueología de Fuego Patagonia. Levantando piedras, desenterrando huesos... y develando arcanos (pp. 839-852). CEQUA.

Cassiodoro, G., Guichón, F., y Re, A. (2019). Diseños sobre soportes móviles y comunicación en el centro-oeste de Santa Cruz durante el Holoceno tardío. En J. Gómez Otero, A. Svoboda y A. Banegas (Eds.), Arqueología de la Patagonia: el Pasado en las Arenas (pp. 29-40). Altuna Impresores.

Chaile, C., Goñi, R., y Cassiodoro, G. (2020). Uso de cerámica en cazadores-recolectores del centro-oeste de la provincia de Santa Cruz (Patagonia Argentina). En V. Schuster y A.
E. Pérez (Eds.), Cerámica Arqueológica Patagonia (pp. 59-76). Fundación de Historia Natural Félix de Azara.

Charlin, J., Borrero, L., y Gómez Otero, J. (2018). Las primeras ocupaciones en el alero Potrok Aike 1 (Santa Cruz): evidencias líticas, óseas y paleoambientales. Revista del Museo de Antropología, 11(2), 23-38. http://doi. org/10.31048/1852.4826.v11.n2.19442

Cirigliano, N. A., Montenegro, T., Franco, N. V., Brook, G. A., y Cherkinsky, A. (2019). Hallazgos cerámicos y su cronología en el área comprendida entre el extremo sur del Macizo del Deseado y la cuenca del río Santa Cruz (Patagonia sur, Argentina). Magallania, 47(2), 132-149. http://doi.org/10.4067/S0718-22442019000200132

Convención Nacional de Antropología (1966). Primera Convención Nacional de Antropología, Primera parte. Universidad Nacional de Córdoba.

Corbella, H. (2002). El campo volcánico-tectónico de Pali Aike. En M. J. Haller (Ed.), Geología y recursos naturales de Santa Cruz. Relatorio del XV Congreso Geológico Argentino. El Calafate (pp. 285-301). Asociación Geológica Argentina.

D’Orazio, M., Agostini, S., Mazzarini, F., Innocenti, F., Manetti, P., Haller, M. J., y Lahsen, A. (2000). The Pali Aike Volcanic Field, Patagonia: slab-window magmatism near the tip of South America. Tectonophysics, 321, 407427. http://doi.org/10.1016/S0040-1951(00)00082-2

Frére, M. M., González, M. I., Chan, D., y Flores, M. C. (2012). Petrografía de la alfarería arqueológica del río Salado Bonaerense. Comechingonia Virtual, 16(2), 115-137. http://doi.org/10.37603/2250.7728.v16.n2.17992

Gómez Otero, J. (1987). Posición estratigráfica particular de puntas de los períodos IV y V de Bird en el alero Potrok-Aike (Santa Cruz). En Primeras Jornadas de Arqueología de la Patagonia (pp. 125-130). Dirección de Cultura de la Provincia de Chubut.

Gómez Otero, J. (1989-90). Cazadores tardíos en la zona fronteriza del paralelo 52 Sur: El paraje de Juni Aike. Anales del Instituto de la Patagonia, 19, 47-71.

Gómez Otero, J., y Barbería, E. (1982). La "casa grande" de Hill Station. Testimonio de toda una época. Mundillo Ameghiniano, 8 y 9, 13-17 y 13-16.

Gradin, C. J. (2000). Más allá y más acá del Río Santa Cruz. Nuevo Offset.

L'Heureux, G. L., Borrazzo, K., y Charlin, J. (2021a). Cronología de las ocupaciones humanas en el campo volcánico Pali Aike: aportes desde el interfluvio Gallegos-Chico y valle medio del río Chico (Santa Cruz, Argentina). Enviado a Revista Magallania. Ms.

L'Heureux, G. L., Ozán, I., y Tripaldi, A. (2021b). Sedimentos, geoquímica y huesos: tafonomía actualística en el interfluvio de los ríos Gallegos y Chico, Patagonia, 
Argentina. 2nd Workshop on Actualistic Taphonomy (p. 26). Centro de Estudos Costeiros, Limnológicos e Marinhos, CECLIMAR, UFRGS Litoral Norte. http:// doi.org/10.5281/zenodo.511454

Manzi, L., y Carballo Marina, F. (2012). Manifestaciones rupestres en el campo volcánico Pali Aike (cuenca del río Gallegos, Santa Cruz, Argentina). Magallania, 40(1), 283-302. http://doi.org/10.4067/S0718-22442012000100017

Manzi, L., Ozán, I., Charlin, J., Pallo, C., Cirigliano, N., Borrazzo, K., y L'Heureux, G. (2019). Pinturas y grabados rupestres en el interfluvio Gallegos-Chico (Provincia de Santa Cruz, Argentina): ampliando límites espaciales y temporales. En J. Gómez Otero, A. Svoboda y A. Banegas (Eds.), Arqueología de la Patagonia: el Pasado en las Arenas (pp. 661-670). Altuna Impresores.

Martin, F., y San Román, M. (2010). Explorando la variabilidad del registro arqueológico y tafonómico en Pali-Aike (Chile) a través de la búsqueda de registros pleistocénicos a cielo abierto. Magallania, 38(1), 199-214. http://doi. org/10.4067/S0718-22442010000100012

Martinic B., M. (1984). Noticia histórica sobre una prospección arqueológica realizada por Junius Bird a lo largo de la costa nororiental del estrecho de Magallanes. Anales del Instituto de la Patagonia, 15, 43-45.

Martinic B., M. (1993-94). Jorge C. Schythe, coleccionista etnográfico. Anales del Instituto de la Patagonia, 22, 5-31.

Martinic B., M. (1995). Los Aónikenk, historia y cultura. Ediciones Universidad de Magallanes.

Martinic B., M., y Prieto, A. I. (1998). La cerámica entre los Aonikenk. Anales del Instituto de la Patagonia, 26, 77-82.

Massone, M. (1981). Arqueología de la región volcánica de Pali Aike (Patagonia meridional chilena). Anales del Instituto de la Patagonia, 12, 95-124.

Massone, M. (1984). Los paraderos tehuelches y prototehuelches en la costa del estrecho de Magallanes. Anales del Instituto de la Patagonia, 15, 27-42.

Massone, M. (1989-90). Investigaciones arqueológicas en la Laguna Thomas Gould (1980-1982). Anales del Instituto de la Patagonia, 19, 87-99.

Massone, M., y Hidalgo, E. (1981). Investigaciones arqueológicas en alero Pali Aike 2. Anales del Instituto de la Patagonia, 12, 125-140.

Mena, F. L., y Jackson, D. S. (1991). Tecnología y subsistencia en Alero Entrada Baker Región de Aisén, Chile. Anales del Instituto de la Patagonia, 20, 169-203.

Mena, F., y Lucero, V. (2004). En torno a las últimas poblaciones indígenas de la cordillera centro-patagónica. Estudio comparado de tres valles de Aysén oriental (Chile). En M. T. Civalero, P. M. Fernández y A. G. Guráieb (Comps.), Contra viento y marea, arqueología de la Patagonia (pp. 643-657). INAPL.
Moreno, J. E., y Videla, B. (2008). Rastreando ausencias: la hipótesis del abandono del uso de los recursos marinos en el momento ecuestre en la Patagonia continental. Magallania, 36(2), 91-104. http://doi.org/10.4067/ S0718-22442008000200006

Munsell Color (1992). Munsell Soil Color Charts. Revised Edition. Macbeth - Division of Kollmorgen Instruments Corpsan.

Oriolo, O., Ozán, I. L., Schmidt, B. C., Charlin, J. E., Manzi, L. M., y Techmer, K. (2019). Basalt weathering as the key to understand the past human use of hematitebased pigments in southernmost Patagonia. Journal of South American Earth Sciences 102376. http:// doi.org/10.1016/j.jsames.2019.102376

Orton, C., Tyers, P., y Vince, A. (1997). La Cerámica en Arqueología. Crítica.

Outes, F. (1904). La alfarería indígena de Patagonia. Anales del Museo Nacional de Buenos Aires, 11(4), 33-41.

Panza, J., y Sacomani, L. (2015). Hojas Geológicas 5169-III y 5169-IV Río Gallegos y Cabo Buen Tiempo, Provincia de Santa Cruz, Boletín 413. SEGEMAR.

Powers, M. C. (1953). A new roundness scale for sedimentary particles. Journal of Sedimentary Petrology, 23, 117119. http://doi.org/ 10.1306/D4269567-2B26-11D7$8648000102 \mathrm{C} 1865 \mathrm{D}$

Priegue, C. N. (2007). En Memoria de los Abuelos. Historia de Vida de Luisa Pascual, Tehuelche. Publitek.

Prieto, A. (1984). Hallazgo de un colgante decorado en Morro Chico (Magallanes). Anales del Instituto de la Patagonia, 14, 59-61.

Roumec, A., Zubimendi, M., y Ciampagna, L. (2020). Estudio de la cerámica de la costa norte de Santa Cruz: análisis iniciales de distribución y caracterización tecnomorfológica. En V. Schuster y A. E. Pérez (Eds.), Cerámica Arqueológica Patagonia (pp. 17-34). Fundación de Historia Natural Félix de Azara.

Shepard, A. (1956). Ceramics for Archaeologist. Carnegie Institution Publications.

Skibo, J. M. (1987). Fluvial sherd abrasion and the interpretation of surface remains on southwestern bajadas. Southwestern North American Archaeologist, 8(2), 125-141. http:// doi.org/10.2190/0J02-23DB-34HV-2PFV

Trola, V., y Ciampagna, L. (2011). Primeros análisis de tiestos de cerámica en la Costa Norte de Santa Cruz. En A. F. Zangrando, R. Barberena, A. Gil y G. Neme (Eds.), Libro de Resúmenes de las VIII Jornadas de Arqueología de la Patagonia (p. 78). Museo de Historia Natural de San Rafael.

Zilio, L., Buus, S.A., y Hammond, H. (2018). La colección arqueológica "Pedro Dade" del Museo de La Plata. Revista del Museo de La Plata, 3(2), 368-392. 